\title{
Change in phenotypic plasticity of a morphological defence in Daphnia galeata (Crustacea: Cladocera) in a selection experiment
}

\author{
Shigeto ODA*, Takayuki HANAZATO ${ }^{1)}$ and Koichi FUJII') \\ National Institute for Environmental Studies, Research Centre for Environmental Risk, Onogawa 16-2, Tsukuba, Ibaraki 305-8506, \\ Japan \\ ${ }^{1)}$ Research and Education Centre for Inlandwater Environment, Shinshu University, 5-2-4 Kogandori, Suwa 392-0027, Japan \\ ${ }^{2)}$ Institute of Biological Sciences, University of Tsukuba, Ibaraki 305-0006, Japan \\ *e-mail corresponding author: oda.shigeto@nies.go.jp
}

\begin{abstract}
Some water fleas Daphnia change their head morphology to reduce predation risk in response to chemical substances (kairomones) released from larvae of the invertebrate predator Chaoborus (Insecta: Diptera). We tested for evidence of the costs associated with elongation of the head spine in Daphnia galeata and the consequences of these costs on the inducibility of head spine elongation in predictable and unpredictable environments. We exposed $\mathrm{D}$. galeata in outdoor experimental ponds to conditions under which predation pressure by Chaoborus larvae and the concentration of kairomones from this predator were controlled for about 70 days. In the laboratory, we then used Daphnia clones collected from the outdoor ponds to investigate the inducibility of head spine formation in response to Chaoborus kairomones.

The inducibility of head spine formation increased in $\mathrm{D}$. galeata from the ponds that had contained both predators and kairomones, whereas in water fleas from the ponds containing only kairomones the plasticity (inducibility) of head spine formation decreased compared with that in the control ponds. These results suggest that the production of a defensive head spine, its phenotypic plasticity, or both entail some costs. Contrary to our predictions, exposure to Chaoborus kairomones in the laboratory resulted in head lengths that were not significantly different among any of the clones from the three outdoor treatments.

We found no evidence for costs associated with head spine elongation in terms of fecundity, time to maturity, or intrinsic rate of natural population increase. Average within-clone partial correlations calculated for head length and intrinsic rate of natural population increase, corrected for body length, were not significantly negative, indicating no cost of defence. This was probably because food conditions in the laboratory were so good that the costs of defence could not be detected. Furthermore, community-level changes, such as changes in food conditions caused by manipulation of the predation regime in the outdoor ponds, might have worked as selection pressures and confounded the results.
\end{abstract}

Key words: Chaoborus flavicans, cost, induced defence, kairomone

\section{INTRODUCTION}

Phenotypic plasticity, the potential of a single genotype to produce different phenotypes in response to changes in environmental conditions (Bradshaw 1965), is an effective adaptation to variable environments when environmental cues are available. Phenotypic plasticity has been studied vigorously, both empirically and theoretically (e.g., for reviews see Scheiner 1993; Schlichting \& Pigliucci 1998).

Inducible defences in organisms, which include the induction of alterations in behaviour, chemistry, life history, colour, or morphology in response to cues from consumers or competitors, can be seen as adaptive phenotypic plasticity. In aquatic communities, the release of chemical substances from predators often gives rise to changes in morphology, life-history traits, or behaviour in prey species (reviewed in Havel 1987; Adler \& Harvell 1990; Harvell 1990). These chemicals are generally called 'kairomones', as these induced changes confer benefits to the prey species (Dicke \& Sabelis 1988).

The induced changes in morphology, for example, are beneficial because they decrease the vulnerability of the prey species to predation (Gilbert 1980; Krueger \& Dodson 1981; Harvell 1984; Havel \& Dodson 1984; Lively 1986a; Mort 1986; Nilsson et al. 1995; Spitze \& Sadler 1996). If the induced defences have benefits without fitness costs associated with their development, they should be regarded as constitutive or fixed defences rather than phenotypically plastic ones (Lively 1986b; Clark \& Harvell 1992). Therefore, the costs associated with these inducible defences have been looked for as the reason behind the prevalence of inducible defences.

The cost-benefit framework predicts not only the evolution of inducible defences under a predictable environment, but also the dominance of fixed defences or lack of defences in an unpredictable environment (Lively 1986b). Few experimental studies, however, have tested these predictions. In the present study, we demonstrate the validity of the cost-benefit framework in explaining the evolution of inducible defences by manipulating environmental cues (kairomones and predation) in an experimental system of zooplankton and predators.

The freshwater crustacean Daphnia is a major con- 
stituent of zooplankton communities. These species alter their morphology (formation or elongation of a head spine, helmet, or neck-teeth) in response to chemical substances released by invertebrate predators such as backswimmers (Grant \& Bayly 1981) or larvae of the phantom midge Chaoborus (Insecta: Diptera) (Krueger \& Dodson 1981; Hanazato 1991) or by predatory fishes (Tollrian 1994). They also change their life-history traits (Dodson \& Havel 1988; Stibor 1992) and exhibit diel vertical migration (Dodson 1988; De Meester 1993, 1996) in the presence of chemical cues from fish or invertebrate predators. They usually exhibit cyclical parthenogenesis, by which both asexual and sexual reproduction exist in the life cycle. Asexual reproduction, which predominates most of the time, enables direct description of within-clone variation as a reaction norm; this variation is defined as the difference between the mean phenotypic values expressed in several environments by clonal replicates. Thus, with their short generation time and ease of manipulation, Daphnia are among the most favourable organisms for studying the evolution of phenotypic plasticity of morphology, life history, and behaviour as mediated by chemical cues from predators (e.g., Tollrian \& Dodson 1998).

Here, we controlled predation and predator kairomones in a population of Daphnia galeata in a zooplankton community established in outdoor experimental ponds. We searched for evidence of the costs associated with elongation of the head spine and the consequences of these costs in terms of the inducibility of head spine formation in D. galeata under predictable and unpredictable environments.

We assumed that the induced morphological defence in Daphnia (i.e., development of a head spine in response to predator kairomones) was beneficial to Daphnia in the presence of Chaoborus at the expense of a decrease in fitness. We also assumed that there was a genetically based variability in the inducibility of head spine development, and that this variability was associated with fitness. Three predictions were derived from these assumptions: (1) in the environment with predatory Chaoborus larvae and kairomones, Daphnia clones that were sensitive to kairomones would increase in number because the induction of spine formation was beneficial over the costs of defence in the presence of predators; (2) in the environment containing only kairomones without predators, Daphnia clones with less sensitivity to kairomones would be favoured because of the costs associated with elongation of the head spine; and (3) in the environment with neither predators nor kairomones, a wide variety of sensitivity to kairomones would be observed, because the ability to form a head spine would be obscured and not exposed to selection.

\section{MATERIALS AND METHODS}

\subsection{Outdoor experiment}

Nine outdoor concrete ponds ( 1.5 × 2 × $0.7 \mathrm{~m}$ deep) were used. The ponds were lined with polyethylene film to prevent any influence from previous experiments. The ponds were filled with ground water to a depth of $0.5 \mathrm{~m}$ (water volume $1500 \mathrm{l}$ ) before the experiment. To establish zooplankton communities, about $7.7 \mathrm{~kg}$ of bottom mud from the eutrophic Lake Nakanuma (Japan: $35^{\circ} 53^{\prime} \mathrm{N}, 140^{\circ} 09^{\prime} \mathrm{E}$ ), which contained the resting stages of phytoplankton and zooplankton, was introduced into each pond on 14 September 1997. Before the introduction, the mud was sieved with a $0.59-\mathrm{mm}$ mesh net to eliminate larvae of the invertebrate predator Chaoborus flavicans. Daphnia galeata and Daphnia ambigua are major cladocerans in Lake Nakanuma, which is inhabited by planktivorous fish (e.g., bluegill sunfish, Lepomis macrochirus) and $C$. flavicans larvae. The bottom mud was collected from the lake with an Ekman-Birge dredge on 4 September 1997, and it was kept in the dark in a temperature-controlled room $\left(10^{\circ} \mathrm{C}\right)$ until the start of the experiment. The surfaces of all ponds were covered with nets (mesh size $1.5 \mathrm{~mm}$ ) so that $C$. flavicans adults could not lay eggs in the ponds.

There were three 'predation' ponds. They received C. flavicans egg masses collected from a nearby pond containing abundant $C$. flavicans larvae. The Daphnia in these ponds were thus subjected to both predation by $C$. flavicans larvae and the chemical effects of $C$. flavicans kairomones. Introduction of $C$. flavicans egg masses was started on 22 September (day 9 of the experiment) and was repeated two or three times a week to compensate for the loss of larvae through emergence as adults. The three 'kairomone' ponds received water containing the kairomones released from $C$. flavicans larvae. This water was supplied daily to the three ponds from 3 October until the end of the experiment (5 December) and was prepared as follows. To supply each kairomone pond, about 1000 fourth-instar larvae of C. flavicans collected from a nearby pond were kept in a 45-1 fibreglass-reinforced plastic container and were fed Daphnia rosea and Moina macrocopa. All of the water (about 40 litres) from the container was sprinkled over the pond once a day after being filtered through a net (mesh size $20 \mu \mathrm{m}$ ) that prevented the passage of most zooplankton. The containers with the remaining $C$. flavicans larvae were refilled with water from the kairomone ponds after most of the zooplankton had been removed from these ponds by sieving with a net (mesh size $20 \mu \mathrm{m}$ ). The three remaining ponds were assigned as 'controls' and contained Daphia but no predators and no kairomones.

Water was sampled from the centre of each pond after the water column had been mixed gently to homogenize the distribution of zooplankton. Sampling was conducted twice a week from 18 September until 5 December using a Kitahara sampler (Rigosha \& Co., 
Ltd., Saitama, Japan), which isolated a column of water (1.3 1) from the surface to $10 \mathrm{~cm}$ above the sediment. Zooplankton were collected by passing two samples of water (2.6 1) through a 40- $\mu \mathrm{m}$ mesh net and then fixed with sucrose formalin (Haney \& Hall 1973). The number of zooplankton was counted under a binocular microscope. Chaoborus flavicans larvae were collected by towing a plankton net (mesh size $94 \mu \mathrm{m}$, diameter 20 $\mathrm{cm}$ ) vertically from the bottom to the surface of the pond, and then preserved in sucrose formalin.

The chlorophyll $a$ concentration in the water was determined by the method of Talling \& Driver (1963, cited in Vollenweider 1974). On each sampling day, the water temperature, dissolved oxygen concentration (DO), and $\mathrm{pH}$ in each pond were measured with a DO meter (YSI Model 55, YSI Hydrodata Ltd., Hertfordshire, UK) and a Beckman F44 pH meter (Beckman Coulter, Inc., CA, USA).

Samples for morphological measurement were collected on day 41 of the experiment (24 October) by taking a bucketful of water (about $20 \mathrm{~L}$ ) from the centre of each experimental pond after gently mixing the water column. Samples were fixed with sucrose formalin, and the head length (from the eye to the helmet tip) and body length (from the eye to the base of the tailspine) of $D$. galeata were measured with a profile projector (Topcon, Model PP70, TOPCON CORPORATION, Tokyo, Japan).

\subsection{Laboratory experiment}

Four individual Daphnia, each hereafter called a 'clone', were collected from each pond on 6 December and cultured separately. Each clone was maintained in a $200 \mathrm{~mL}$-beaker of dechlorinated tap water containing $1 \times 10^{6}$ cells ml-1 of Chlorella vulgaris as food. Each day a sufficient quantity of algae was supplied to maintain the initial algal concentration. Every 3 days 10 to 15 animals of each clone from each pond were transferred by pipette to fresh medium. For three generations before the laboratory experiment, the numbers of each clone in the culture medium were maintained at 20 to 30 individuals per beaker to lessen possible maternal effects (Lynch 1985) and acclimatize the clones to the environment.

'Kairomone water', which contained chemical substances from $C$. flavicans larvae, was prepared as follows. Fourth-instar larvae of $C$. flavicans were collected from a pond near the experimental ponds and were reared in aged tap water at a density of 30 individuals $1^{-1}$ for 2 days in a temperature-controlled room $\left(22 \pm 1^{\circ} \mathrm{C}\right)$. Enough cladocerans ( $M$. macrocopa) were provided daily as food. After the larvae had been reared, the water was filtered through a Whatman GF/C filter to remove any particles $>1 \mu \mathrm{m}$ in diameter and then stored in a freezer at $-30{ }^{\circ} \mathrm{C}$, under which conditions the chemicals from Chaoborus larvae are stable for at least 6 months (Hebert \& Grewe 1985). The frozen water was thawed at room temperature before the experiment and used as the kairomone water rearing medium, after it had been diluted to one-third with dechlorinated tap water. Dechlorinated tap water, in which Chaoborus larvae had never been reared, was used as the 'control water'.

The effect of the predation regimes in the outdoor experiment on the degree of phenotypic plasticity was expressed as the difference in the slopes connecting the mean head lengths of clones from the three different outdoor treatments (control, Chaoborus, and kairomone) reared in two different culture media (control water and kairomone water) in the laboratory. At the beginning of the laboratory part of the experiment, adult Daphnia that had eggs in their brood chambers were transferred to two beakers filled with $100 \mathrm{~mL}$ of the experimental medium (control water or kairomone water). The population densities were kept to about 10 per beaker. Chlorella was provided as food at a concentration of $1 \times 10^{6}$ cells $\mathrm{ml}^{-1}$. After exposure to the experimental medium for 24 hours, the adults were transferred to fresh medium and kept for a further $14 \mathrm{~h}$. Three neonates born in each beaker within this 14 -h period and destined for use as replicates for the measurement of clone values were picked up by pipette and individually put into test tubes containing $20 \mathrm{~mL}$ of experimental medium in which Chlorella was suspended at a density of $1 \times 10^{6}$ cells $\mathrm{mL}^{-1}$ as food. The neonates used to measure morphology and life-history traits were taken from the mother's third (or later) clutch. The test tubes were placed in an incubator temperature-controlled at $23 \pm$ $0.5^{\circ} \mathrm{C}$ and with a photoperiod of $14: 10 \mathrm{~h}$ light:dark.

Individuals were videotaped under a microscope and the images were measured afterwards with the aid of a Himawari video digitizing system (Library \& Co., Ltd., Tokyo, Japan). Several morphological and life-history traits were measured. Head length and body length were measured at the second juvenile instar and at the first and second clutches. Fecundity (total number of eggs carried at the first and second reproductions) was also recorded. Time to maturity was determined by monitoring the individuals daily. The animal was transferred to fresh rearing medium immediately after measurement. The intrinsic rate of natural population increase was calculated from the information on the first two clutches by using the stable-age (Euler's) equation. As the time of release of neonates was not observed in the case of the second clutch, we regarded it as 2 days after eggs had been seen in the brood chamber, because in the first clutch $98.4 \%$ of neonates had been released from the brood chambers within 2 days.

\subsection{Statistical analyses}

The morphological changes in D. galeata in the outdoor experiment were analysed as the difference in the allometric relationship between head length and body length after natural log-transformation. The three ponds 
in each treatment (control, Chaoborus, and kairomone) were nested in the field (hereafter called 'pond effect'), and the nested ANCOVAs were performed with body length as a covariate using PROC GLM and PROC REG in SAS (SAS Institute 1988). Differences in regression coefficients were tested by the Tukey-Kramer method (Sokal \& Rohlf 1995).

The morphological and life-history traits of animals measured in the laboratory experiment were analysed by two-way nested ANOVAs and ANCOVAs with or without repeated measurements. The full models for ANOVAs and ANCOVAs with morphological and lifehistory traits were as follows. Outdoor treatment (predation effect: control, Chaoborus, and kairomone) and laboratory treatment (medium effect: control water and kairomone water) were treated as fixed effects. The three ponds nested in each treatment in the outdoor experiment (pond effect) and the four clones nested in each pond (clone effect) were considered as random effects. Morphological traits were measured for the same individual several times (stage effect), that is, at the second instar, at the first adult instar, and at the second adult instar. The head length of D. galeata is largely dependent on body length. Therefore, to compare the phenotypic plasticity of head length independently of body length, nested ANCOVAs were used, with body length as a covariate. Head length and body length were analysed after natural log-transformation. Statistical analyses of all traits were performed with PROC MIXED in SAS (Littell et al. 1996).

For further analyses of significant interaction effects between the predation effect and the medium effect, two contrasting interaction effects were tested (Milliken \& Johnson 1984). The null hypotheses for the two contrasts were as follows. First, the plasticities of D. galeata from the Chaoborus ponds and from the control ponds were the same. The second one was that the plasticities of individuals from the kairomone ponds and those from the control ponds were the same.

Because the age at which Daphnia has eggs for the first and the second clutches differs among clones, we also analysed head length and body length measured at the second and fifth instars (At these instars the head and body have both experienced the same number of molts).

The costs of an inducible defence include both the costs of defence and the costs of plasticity. The defensive costs of head length were estimated as among-individual, within-clone correlations between head length and intrinsic rate of natural increase using PROC NESTED in SAS as in Scheiner \& Berrigan (1998). Correlations of the residuals, after the removal of any genetic (among-clone) effect, measured the costs of defence averaged across clones. As body length has a strong influence on both head length and intrinsic rate of natural population increase, partial correlations between them, with body length held constant, were calculated to examine the costs of defence. The costs of defence were expressed as negative partial correlations between head length and intrinsic rate of natural increase. Head length and body length were natural log-transformed before the analysis. We estimated the correlations separately for each medium level at the second instar, at the first and second reproductions, and at the fifth instar.

\section{RESULTS}

\subsection{Population dynamics of Daphnia galeata and Chaoborus flavicans in the experimental ponds}

The water temperature in the experimental ponds, which was $22{ }^{\circ} \mathrm{C}$ at the start of the experiment, had declined steadily to $7^{\circ} \mathrm{C}$ by the end of the experiment. The difference in water temperature among the ponds was usually less than $2{ }^{\circ} \mathrm{C}$. DO in the ponds first increased after the start of the experiment, gradually declined towards the middle, and then increased again near the end of the experiment. It ranged from 6.7 to $13.7 \mathrm{mg} \mathrm{l}^{-1}$. The $\mathrm{pH}$ increased first, stayed constant at about 9.3 for a month, and then declined gradually towards the end of the experiment. It ranged from 7.6 to 9.4. The chlorophyll $a$ concentration increased rapidly to a maximum of about 11.5 to $19.1 \mu \mathrm{g} \mathrm{l}^{-1}$ in the nine ponds just after the introduction of the lake mud and was followed by a sharp decline; it then fluctuated between 0.06 and $6.4 \mu \mathrm{g} \mathrm{l}^{-1}$, except in the Chaoborus ponds, where it stayed at a constant value of about $1 \mu \mathrm{g} \mathrm{l}^{-1}$ after the first sharp increase.

The number of $D$. galeata in the control ponds increased to 70 to 110 individuals $1^{-1}$ by day 48 of the experiment ( 31 October); this was followed by several fluctuations (Fig. 1a).

In the Chaoborus ponds, the density of $C$. flavicans at the third and fourth instars (at which ages the larvae have the largest influence on the mortality of D. galeata) fluctuated between 0.5 and 2 individuals $1^{-1}$. Daphnia galeata in the Chaoborus ponds stayed at a relatively low density during the experiment compared with the controls, presumably because of $C$. flavicans predation (Fig. 1b).

Throughout the experiment, D. galeata in the kairomone ponds tended to stay at high densities relative to those in the control ponds, probably because the daily addition of kairomones included nutrients which may have stimulated phytoplankton growth (Fig. 1c).

\subsection{Morphological changes in Daphnia galeata in the experimental ponds}

The experimental treatments in the outdoor ponds changed the allometric relationships between head length and body length in D. galeata (Tabs 1a and b). There was a significant interaction effect between the experimental treatment and the covariate of body length: that is, the three treatments gave different slopes (ANCOVA; predation $\mathrm{x}$ body length, $F_{2,252}=8.24, \mathrm{P}=$ 0.0003). The steeper slope for the allometric relation- 

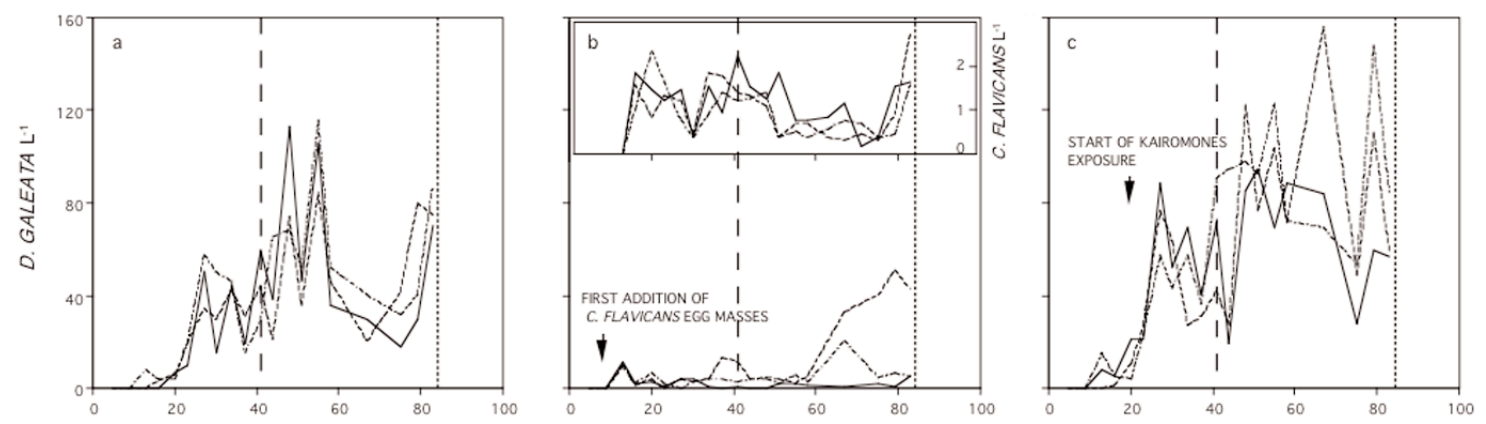

DAYS FROM START OF EXPERIMENT

Fig. 1. Population dynamics of Daphnia galeata in experimental ponds: (a) control ponds, (b) Chaoborus ponds, (c) kairomone ponds. Three lines for each panel show replicates. Broken and dotted vertical lines indicate sampling date for morphological measurement and sampling date of individuals for the laboratory experiment, respectively. Inset in (b) shows the density of Chaoborus flavicans larvae at the third and fourth instar.

ship between head length and body length in the Chaoborus ponds than in the control and the kairomone ponds (Tab. 1a), combined with the statistically significant contrast between the control and Chaoborus $\left(F_{1,252}\right.$ $=8.65, \mathrm{P}=0.0036$ ), indicate that $D$. galeata in the Chaoborus ponds had a longer head relative to its body length than did those in the control and kairomone ponds. We considered this difference in slopes to be caused by elongation of the head spine in response to Chaoborus kairomones and by a heavier mortality rate of individuals with less elongated heads in the Chaoborus ponds. Similarly, in the kairomone ponds, $D$. galeata altered its head morphology, having a longer head spine relative to its body owing to the presence of kairomones released from $C$. flavicans larvae (Tab. $1 \mathrm{~b}$; predation $F_{1,176}=30.90, \mathrm{P}=0.0001$ ), compared with $D$. galeata in the control ponds.

\subsection{Effects of treatment in the experimental ponds on the phenotypic plasticity of head length measured in the laboratory experiment}

Differences in phenotypic plasticity caused by treatments in the outdoor experiment can be expressed by

Tab. 1. Results of regression analysis and analysis of covariance of allometric relationships between head length and body length of Daphnia galeata on day 41 in the experimental ponds. Data were natural log-transformed before analysis. Measurements for the three replicate ponds were pooled because the pond effect was not statistically significant. (a) Estimated regression coefficients "b". Significant differences in slopes were determined by the TukeyKramer method and are shown in bold. Underlined value was marginally significant. (b) Results of an analysis of covariance fitting a common slope for the control ponds and the kairomone ponds.

\begin{tabular}{lllllll}
\hline & \multicolumn{2}{c}{ Slope } & & \multicolumn{3}{c}{ Difference in slopes } \\
\cline { 5 - 7 } \cline { 5 - 6 } & Predation & Estimate & & $\mathrm{b}_{\text {Control }}$ & $\mathrm{b}_{\text {Chaoborus }}$ & $\mathrm{b}_{\text {Kairomone }}$ \\
\hline Control & $\mathrm{b}_{\text {Control }}$ & 0.363 & & - & - & - \\
Chaoborus & $\mathrm{b}_{\text {Chaoborus }}$ & 0.675 & & $\mathbf{0 . 3 1 1}$ & - & - \\
Kairomone & $\mathrm{b}_{\text {Kairomone }}$ & 0.493 & & 0.129 & $\underline{0.182}$ & - \\
\hline
\end{tabular}

\begin{tabular}{lllll}
\hline Source & df & MS & $F$-value & $P$-value \\
\hline Predation & 1 & 0.1190 & 30.9 & 0.0001 \\
Body length & 1 & 0.3701 & 96.1 & 0.0001 \\
Residual & 176 & 0.0039 & & \\
\hline
\end{tabular}


two-way ANOVA as a significant predation $\mathrm{x}$ medium interaction effect. When head length was analysed by repeated measures of ANCOVA for three developmental stages (second instar, ages at the first and second reproductions), a significant interaction effect of predation $\mathrm{x}$ medium was found (Fig. 2a, Tab. 2a). We tested the contrasts in the interaction effect further, and we found that D. galeata from the Chaoborus ponds had a significantly steeper reaction norm than those from the control ponds, whereas the reaction norms for animals from the kairomone ponds and the control ponds were not significantly different (Tab. 2a). Similarly, ANCOVA with
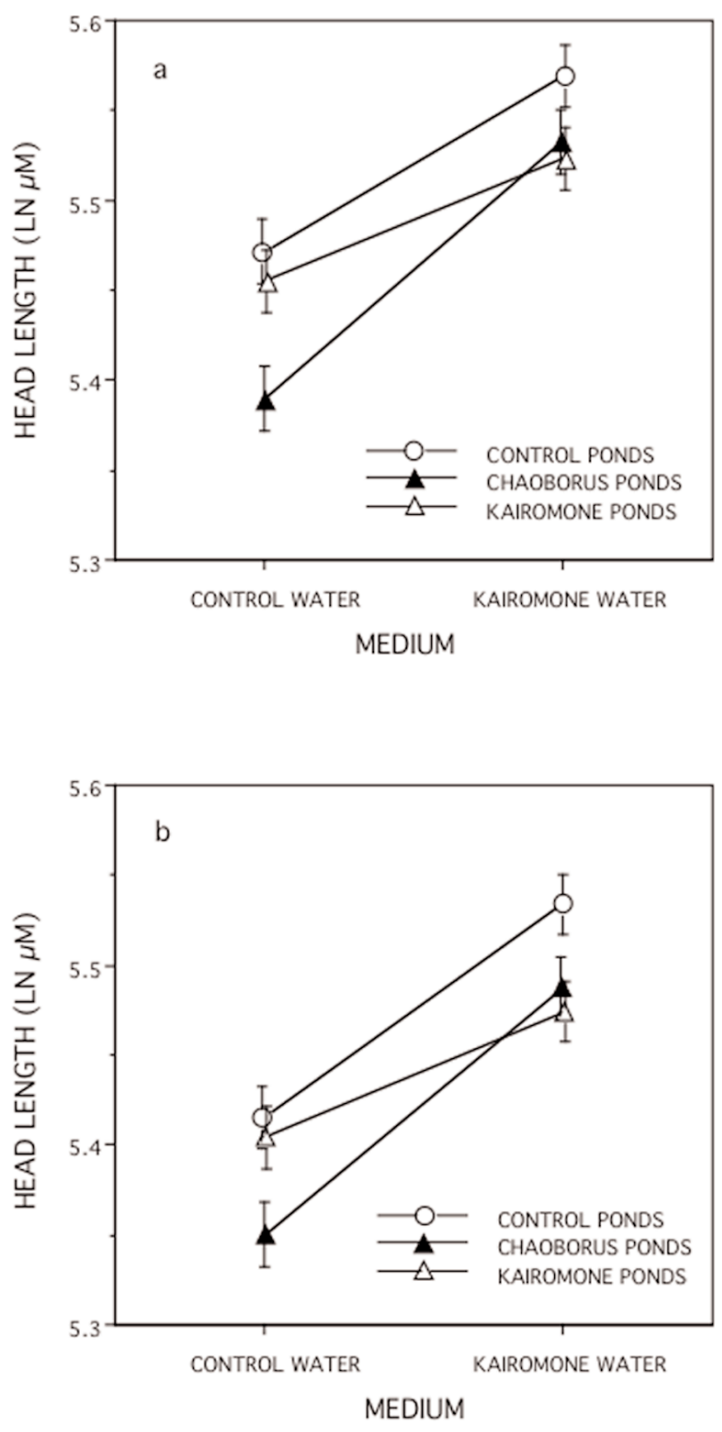

Fig. 2. Reaction norms of Daphnia galeata, describing the pattern of head length in response to Chaoborus kairomones for the three experimental treatments in the outdoor ponds. Reaction norms are shown as those of least square means; head length was adjusted to a grand mean of body length and averaged (a) for three developmental stages (at the second instar, and at the first and second reproductions) or (b) for two ages (at the second instar and at the fifth instar). Error bars indicate one standard error.
Tab. 2. Results of mixed model analyses of covariance with repeated measurements of head length plasticity data, using PROC MIXED for (a) three developmental stages (at the second instar, and at the first and second reproductions) or (b) two ages (at the second instar and at the fifth instar). Body length was included in the model as a covariate. Each variance component is followed by one standard error, as estimated by asymptotic theory. $P$-values were obtained by log-likelihoodratio tests. Significance of fixed effects were tested by $F$-test. $P$-value with asterisks indicate contrasts that were significant at a level adjusted by the Dunn-Sidák method to maintain the experiment-wise error rate at $\mathrm{P}=0.05$.

Table 2a

\begin{tabular}{lrrr}
\hline Source & Estimate & SE P-value \\
\hline Random effects & 0.0022 & 0.0008 & $<0.0001$ \\
Clone & 0.0011 & 0.0004 & $<0.0001$ \\
Clone X Stage & 0 & \\
Clone X Medium & F-value & P-value \\
\hline Source & & \\
\hline Fixed effects & & \\
Predation & 3.28 & 0.1367 \\
(Control-Chaoborus) in Kairomone water & 2.04 & 0.2040 \\
(Control-Kairomone) in Kairomone water & 3.37 & 0.1220 \\
Medium & 160.7 & 0.0001 \\
Body length & 30.89 & 0.0001 \\
Stage & 1.19 & 0.3176 \\
Predation X Medium & 7.00 & 0.0012 \\
Control - Chaoborus & 5.20 & $0.0240 *$ \\
Control - Kairomone & 2.19 & 0.1410 \\
Predation X Stage & 1.04 & 0.4118 \\
Medium X Stage & 10.3 & 0.0001 \\
Predation X Medium X Stage & 0.85 & 0.4986 \\
\hline & &
\end{tabular}

Table 2b

\begin{tabular}{lrrr}
\hline Source & Estimate & SE P-value \\
\hline Random effects & & & \\
Clone & 0.0015 & 0.0008 & $<0.0001$ \\
Stage X Clone & 0.0019 & 0.0007 & $<0.0001$ \\
Clone X Medium & 0 & \\
& & \\
\hline Source & F-value & P-value \\
\hline Fixed effects & & \\
Predation & & \\
(Control- Chaoborus) in Kairomone water & 3.17 & 0.1317 \\
(Control-Kairomone) in Kairomone water & 3.55 & 0.1032 \\
Medium & 6.06 & 0.0483 \\
Body length & 177.7 & 0.0001 \\
Stage & 47.48 & 0.0001 \\
Predation X Medium & 7.51 & 0.0071 \\
Control - Chaoborus & 6.16 & 0.0027 \\
Control - Kairomone & 0.89 & 0.3474 \\
Predation X Stage & 6.22 & $0.0137 *$ \\
Medium X Stage & 0.63 & 0.5461 \\
Predation X Medium X Stage & 16.7 & 0.0001 \\
& 0.29 & 0.7455 \\
\hline
\end{tabular}


repeated measurements for two stages (second and fifth instars) showed a statistically significant predation $\mathrm{x}$ medium interaction effect (Fig. 2b, Tab. 2b). Further analyses using contrasts, however, contradicted the result obtained from the three stages data. We found that the slope of the reaction norm for D. galeata from the kairomone ponds was significantly less steep than that from the control ponds, and the slopes of the reaction norms from the control ponds and the Chaoborus ponds were not different (Tab. 2b). The problem is that multiple testing by two analyses not independent of each other makes it difficult to interpret such contradicting results. However, because we wanted to be as liberal as possible in our search for a significant predation $\mathrm{x}$ medium interaction effect, we interpreted these results as supporting our predictions.

\subsection{Comparison of head length in kairomone water}

We predicted that when head length measured in the kairomone water in the laboratory was compared among the three treatments in the field experiment, $D$. galeata from the Chaoborus ponds would have the longest heads, followed by medium-sized heads in Daphnia from the control ponds, and the shortest heads in those from the kairomone ponds. Contrary to our predictions, when the Daphnia were exposed to Chaoborus kairomones, head length did not differ among the three predation treatments, except in our analysis of the data from measurements made at the two ages, in which $D$. galeata from the control ponds had a marginally significantly longer head than that from the kairomone ponds $(\mathrm{P}=0.0483$; Fig. 2b, Tab. 2b).

\subsection{Other morphological and life-history traits}

No significant effect on body size at the first reproduction was found, except for the predation $\mathrm{x}$ medium interaction effect, which was marginally significant (Tabs 3 and 4). Clones from the Chaoborus ponds had a tendency to have smaller body lengths in the kairomone water than in the control water at the first reproduction, whereas the two other treatments gave the opposite pattern (Tab. 3). Body length at the second instar showed a similar pattern, but the predation $\mathrm{x}$ medium interaction effect was not significant (Tabs 3 and 4).

Fecundity, as measured by total number of eggs carried at the first and second reproductions, was not affected by the predation regime in the outdoor ponds (Tabs 3 and 4). Fecundity was significantly greater in the kairomone water than in the control water. A statistically significant variance component was observed for the clone effect (Tab. 4). This finding, together with the lack of a significant predation $\mathrm{x}$ medium effect on fecundity, indicates that there were no significant effects on fecundity in terms of the cost of head spine development.

For time to maturity, the predation, medium, and predation $\mathrm{x}$ medium interaction effects were not statistically significant (Tabs 3 and 4). Significant variance components were found for the clone effect and the clone $\mathrm{x}$ medium interaction effect (Tab. 4). No effect indicating evidence for costs associated with elongation of a head spine was found.

The intrinsic rates of natural population increase were compared to see whether there was any fitness cost associated with the formation of a head spine (Tabs 3 and 4). Data analysed by two-way ANOVA showed a statistically significant medium effect and clone effect,

Tab. 3. Means of morphological and life-history traits of Daphnia galeata from three experimental treatments when exposed to two different media in the laboratory. See also Table 4 for significant effects by ANOVA. Body length was natural log-transformed before analysis. Values in parentheses are standard errors.

\begin{tabular}{|c|c|c|c|c|c|c|c|c|c|c|c|c|}
\hline \multirow[b]{3}{*}{ Trait } & \multicolumn{12}{|c|}{ PRE D A T I O N } \\
\hline & \multicolumn{4}{|c|}{ CONTROL } & \multicolumn{4}{|c|}{ CHAOBORUS } & \multicolumn{4}{|c|}{ KAIROMONE } \\
\hline & \multicolumn{2}{|c|}{ Control water } & \multicolumn{2}{|c|}{ Kairomone water } & \multicolumn{2}{|c|}{ Control water } & \multicolumn{2}{|c|}{ Kairomone water } & \multicolumn{2}{|c|}{ Control water } & \multicolumn{2}{|c|}{ Kairomone water } \\
\hline Body length & & & & & & & & & & & & \\
\hline at the $2 \mathrm{nd}$ instar $(\mathrm{mm})$ & 6.617 & $(0.008)$ & 6.623 & $(0.008)$ & 6.615 & $(0.008)$ & 6.606 & $(0.009)$ & 6.610 & $(0.008)$ & 6.614 & $(0.008)$ \\
\hline Body length & & & & & & & & & & & & \\
\hline at $1 \mathrm{st}$ reproduction $(\mathrm{mm})$ & 7.180 & $(0.014)$ & 7.193 & $(0.014)$ & 7.194 & $(0.014)$ & 7.175 & $(0.014)$ & 7.178 & $(0.014)$ & 7.200 & $(0.014)$ \\
\hline Fecundity & 11.89 & $(0.72)$ & 13.35 & $(0.71)$ & 12.22 & $(0.71)$ & 12.85 & $(0.73)$ & 12.66 & $(0.71)$ & 14.2 & $(0.69)$ \\
\hline Time to maturity (d) & 4.88 & $(0.11)$ & 4.82 & $(0.11)$ & 4.94 & $(0.11)$ & 4.76 & $(0.11)$ & 4.81 & $(0.11)$ & 4.80 & $(00.11)$ \\
\hline $\begin{array}{l}\text { Intrinsic rate of } \\
\text { natural increase }\left(\mathrm{d}^{-1}\right)\end{array}$ & 0.584 & $(0.008)$ & 0.598 & $(0.007)$ & 0.585 & $(0.007)$ & 0.597 & $(0.008)$ & 0.591 & $(0.007)$ & 0.612 & $(0.007)$ \\
\hline
\end{tabular}


Tab. 4. Results of mixed model analyses of variance by PROC MIXED of morphological and life-history traits of Daphnia galeata from three experimental treatments exposed to two different media in the laboratory. $F$-values and estimated variances are shown for fixed effects and random effects, respectively. Standard errors were estimated by asymptotic theory. Significances $(\mathrm{P}<0.05)$ of fixed effects and random effects were tested by $F$-test and by log-likelihood-ratio tests, respectively, and are shown in bold. $F$-value with asterisk was marginally significant $(\mathrm{P}=0.076)$.

\begin{tabular}{|c|c|c|c|c|c|c|}
\hline \multirow[b]{2}{*}{ Trait } & \multicolumn{3}{|c|}{ Fixed effects $(F$-value $)$} & \multicolumn{3}{|c|}{ Random effects (variance estimate) } \\
\hline & Predation & Medium & Predation X Medium & Clone & Clone X Medium & SE \\
\hline \multicolumn{7}{|l|}{ Body length } \\
\hline at $2 \mathrm{nd}$ instar $(\mathrm{mm})$ & 0.46 & 0.01 & 0.93 & 0.0004 & 0.00010 & 0.0008 \\
\hline \multicolumn{7}{|l|}{ Body length } \\
\hline at 1st reproduction (mm) & 0.04 & 0.55 & $2.77 *$ & 0.0014 & 0.00022 & 0.0002 \\
\hline Fecundity & 0.6 & 16.38 & 0.99 & 4.4067 & 0 & 3.96 \\
\hline Time to maturity (d) & 0.1 & 1.28 & 0.54 & 0.0451 & 0.04073 & 0.1400 \\
\hline \multicolumn{7}{|l|}{ Intrinsic rate } \\
\hline of natural increase $\left(\mathrm{d}^{-1}\right)$ & 0.92 & 12.54 & 0.32 & 0.0003 & 0.00004 & 0.0008 \\
\hline
\end{tabular}

and the intrinsic rate of natural increase was greater in the kairomone water than in the control water. No significant predation effect was detected. No evidence was found suggesting the existence of costs of developing a longer head spine.

\subsection{Costs of defence as indicated by within-clone correlations}

We could find no direct evidence for the costs of head spine elongation. Daphnia galeata raised in the kairomone water had higher fecundity and intrinsic rates of natural increase than those raised in the control water (Tabs 3 and 4), a finding opposite to our prediction. No significant predation effect or predation $\mathrm{x}$ medium interaction effect for these life-history traits was found.

We also estimated the average within-clone correlations among residuals after removal of the among-clone effect between head length and body length. Partial correlations between head length and intrinsic rate of natural increase, corrected for body length, are presented in Table 5. The partial correlations were calculated for each medium effect (kairomone water or control water). No significant correlation was found for eight coefficients, and only two of the eight coefficients were negative (Tab. 5). Here, again, we could find no evidence for the costs of head spine elongation.

\section{DISCUSSION}

\subsection{Evolution of head spine elongation inducibility}

Treatments in the outdoor experiment changed the phenotypic plasticity or the slope of the reaction norm of morphological defence. Two analyses that were not mutually independent showed different significant differences in the slopes of the reaction norm for head spines for the three outdoor treatments: control, Chaoborus, and kairomone (Figs $2 \mathrm{a}$ and b, Tabs $2 \mathrm{a}$ and b). Although there was a problem with lack of correction for multiple tests, we interpret these results as supportive of our predictions: they indirectly demonstrated the costs of head spine elongation, which works as a morphological defence against predators.

In several studies, patterns of interpopulation genetic differences with respect to response to predator kairomones have been observed. These patterns are consistent with adaptive micro-evolution. In Daphnia, for example, morphologically inducible clones are more likely to be found in ponds with Chaoborus larvae (Parejko \& Dodson 1991) than in those without, and a more plastic response in phototactic behaviour in the presence of fish kairomones has been found in populations of Daphnia with strong predation by fish (De Meester 1996). Our result presents evidence for adaptive micro-evolution of phenotypic plasticity.

However, the result of the comparison of head length (adjusted by body length) when Daphnia were exposed to kairomones in the laboratory was contradictory to our predictions. The observed change of intercepts did not occur in the direction that we expected. That is, the head length of clones from the Chaoborus ponds was not significantly longer than that of clones from the other treatment ponds; furthermore, when Chaoborus pond clones were reared in either the kairomone water or control water in the laboratory, their head length appeared to be shorter than that of the controls (Figs $2 \mathrm{a}$ and $\mathrm{b}$ ). The steeper slopes of the reaction norm of $D$. galeata in the Chaoborus ponds were caused by the fact that it had a much shorter head in the control water than in the kairomone water. This result suggests that interpretation of our results is not simple. One explanation for this contradiction may be that, in the Chaoborus ponds, 
traits other than head length were important. Forces of selection on other traits are probably responsible for the observed changes in the mean value for head spine length in the kairomone water, through genetic correlations between head length and those traits.

Furthermore, although the difference was not significant, clones from the Chaoborus ponds appeared to have a smaller body length at the second instar in response to the kairomone water than those in the control water in the laboratory, whereas clones from the two other treatment ponds were almost the same size, or had slightly larger bodies, in the laboratory kairomone water compared with the control water (Tabs 3 and 4). These findings contradict both the theoretical and experimental predictions. A mathematical model of Taylor \& Gabriel (1992) predicted that it is adaptive for Daphnia to allocate resources to have larger offspring and to delay maturity in the presence of Chaoborus predation. In a selection experiment with $D$. pulex and its predatory Chaoborus larvae, Spitze (1991) found that, after selection by Chaoborus predation, there was in an increase in the number of individuals that were larger at birth, grew faster, matured earlier, and had greater fecundity. On the other hand, the results of some empirical studies are in agreement with ours. Scheiner \& Berrigan (1998) found that juvenile body length was smaller in the presence of an extract of Chaoborus larvae than in its absence.

Regardless of the theoretical and experimental predictions, however, smaller neonate size may be explained in terms of food availability, which is more or less correlated with Chaoborus predation pressure. In general, within the genus Daphnia, neonates that are larger have greater resistance to starvation (Tessier \& Consolatti 1991; Gliwicz \& Guisande 1992). One can predict that optimal neonate size is relatively small under high food availability if the resources available for reproduction are constant (Smith \& Fretwell 1974; but see Boersma 1995). Manipulation of the predation regime in the outdoor ponds could have changed not only the predation and kairomone presence, but also food availability, through community-level interaction. Food availability in the Chaoborus ponds, as indicated by the chlorophyll $a$ concentration, was at a relatively high level compared with that in the other ponds during the outdoor experiment (see Results), probably because of a top-down effect by predators. Thus, under the relatively good food conditions in the Chaoborus ponds, small body size might not have been disadvantageous.

\subsection{Costs of defence}

Because the costs of defence constitute most of the theoretical models for the evolution of inducible defences or phenotypic plasticity, it is important to demonstrate their existence and consequences. Our results support the idea that the shape of the reaction norm of morphological defences is formed by the bal- ance between costs and benefits. In particular, the significantly less steep slopes of the reaction norms observed for D. galeata in the kairomone ponds than in the control ponds suggest the presence of costs associated with head spine formation. Thus, the existence of costs associated with head spine formation was indirectly proved.

However, we could not detect any defensive costs of head spine formation in the analyses using nested ANOVAs. Other life-history traits, such as intrinsic rate of natural increase, total number of eggs carried at the first and second reproductions, or time to maturity (from which we expected to detect evidence of defence costs) provided us with scant evidence. It is possible that the failure to find costs by ANOVA in terms of the significant effects of predation, medium, and predation $\mathrm{x}$ medium were caused by obscuring of the costs by good food availability. Because Scheiner \& Berrigan (1998) could not find direct costs under bad food conditions, we may not have been able to find such costs even if we had kept food availability at a low level. The medium effect could have been be confounded in our experiment not only by kairomones, but also by the nutritious excretions of the predatory $C$. flavicans larvae; these nutrients caused greater fecundity and intrinsic rates of natural increase in D. galeata, a result opposite to that expected if there had been costs. This problem usually makes it difficult to find costs in the laboratory, and few studies have tried to remove this confounding effect (e.g., Tollrian 1995).

\subsection{Costs and limits of phenotypic plasticity}

The genetic change in the inducibility of head spine development in our study could have resulted from mechanisms other than those we expected from natural selection driven by the costs and benefits of having defensive spines. One alternative explanation may be that the cost of phenotypic plasticity could, in itself, have caused the same results. There are several kinds of costs and limits that constrain the evolution of phenotypic plasticity (DeWitt et al. 1998). Although we did not take these plasticity costs into consideration in making our predictions, they could have brought about the same results. For example, maintenance costs (i.e., the costs associated with the maintenance of sensory and regulatory machinery in plastic organisms; DeWitt et al. 1998) could have reduced the slopes of the reaction norm for the clones' head spines in both the control water and kairomone water in our experiment. Clones from the Chaoborus ponds, on the other hand, would have steeper slopes because of the greater benefits of having elongated head spines at the expense of maintenance costs. Thus, maintenance costs can also explain part of our results (the steeper reaction norm observed in the Chaoborus ponds than in the control ponds) without the inclusion of any cost of defence. We do not necessarily need the costs of defence or plasticity costs to explain the above difference between the Chaoborus 
ponds and the control ponds. In contrast, to explain the difference in slopes between the kairomone ponds and the control ponds we do need the costs of defence, and it seems that any cost other than the cost of defence itself cannot explain this difference. It is possible that some costs of phenotypic plasticity might have confounded our results, too, but our experiment was not designed to distinguish these costs. Direct measurements using many genetically different lines (e.g., DeWitt 1998; Scheiner \& Berrigan 1998) would be desirable for detecting these plasticity costs, although a recent study of D. pulex by Scheiner \& Berrigan (1998) failed to detect plasticity costs (maintenance costs and production costs) for kairomone-induced traits.

Because we cannot reject the possibility of the importance of phenotypic plasticity costs and limits as constraints in theoretical phenotypic plasticity evolution, we must be careful in reaching our conclusions. However, it is certain that some costs (mostly the costs of defence, together with plasticity costs, if any) were responsible for the observed differences in phenotypic plasticity between the control ponds and the kairomone ponds.

\subsection{Reliability of kairomones and induced defence}

Riessen (1992) used a demographic model to predict that spatial overlap of prey and predator influences the advantages of induced defences. This shows us that kairomones released from predators work as cues for prey species to determine the risk of predation, and, therefore, that the reliability of kairomones as information is one of the important factors in keeping inducible defences advantageous over fixed defences. Predatory Chaoborus larvae change in number both spatially and temporally (Hanazato 1992), and the corresponding change in kairomone concentration is thought to be reliable information that enables Daphnia to determine the relative predation risk in the environment if the environment is relatively homogeneous, where the spatial distributions of predators and their kairomones are the same. However, in a heterogeneous environment, mismatching of the concentration of kairomones with the degree of predation pressure could bring about disadvantage to induced defences. Thus, plastic organisms in an environment with unreliable information express poor phenotype-environment matching, and the level of information reliability limits the evolution of phenotypic plasticity (Moran 1992; Padilla \& Adolph 1996; DeWitt et al. 1998).

Our population of D. galeata seemed to have a sufficiently large amount of genetic variability of defence inducibility to respond to the experimental treatments in the outdoor ponds. The population that we used in the experiment had a wide range of inducibility of head spine formation in the presence of chemical cues from Chaoborus larvae, because it was derived from resting eggs reproduced sexually. In cyclical parthenogens such as Daphnia, recombination, which usually occurs only in a single sexual generation each year, generates a pulse of genetic variability by exposing hidden genetic variation that builds up under stabilizing selection during clonal reproduction (Lynch \& Gabriel 1983). The large genetic variability may be the result of the release of this hidden genetic variance. In addition, environmental heterogeneity also contributes to the maintenance of genetic variability. Kairomones released from Chaoborus larvae change not only morphology in Daphnia but also their phototactic behaviour. Daphnia galeata, for example, changes its phototactic behaviour in response to kairomones released by Chaoborus larvae (Dodson 1988). This response is thought to cause diel vertical migration, which allows escape from predation by Chaoborus larvae. This behavioural response of $D$. galeata would decrease spatial overlap with Chaoborus larvae, leading to reduced advantage of spine formation (Riessen 1992). In such an environment, a wide variety of inducibility will be observed. Although it has no empirical support, this prediction also suggests that clones more responsive in behaviour to the predator may be less sensitive in terms of morphological change (elongation of the head spine), and vice versa, permitting the coexistence of clones responsive in either behaviour or morphology.

\section{ACKNOWLEDGMENTS}

We thank F. Kasai for valuable comments on this manuscript. We gratefully acknowledge the helpful discussions with T. Mitsunaga and Y. Toquenaga on statistical analysis. Thanks are due to Y. Sugaya for his help in measurement of Daphnia by the Himawari video digitizing system. K. Goka, S. Hatakeyama, and F. Kasai kindly provided access to the National Institute for Environmental Studies. Special thanks are due to K. Suzuki and the staff of Kawakami Farm, Inc., including S. Karube, K. Kawabe, Y. Ogamino, and F. Ohyama, for their help with the field experiment and for preparing algae for the laboratory experiment.

\section{REFERENCES}

Adler, F.R. \& C.D. Harvell. 1990. Inducible defenses, phenotypic variability and biotic environments. Trends Ecol. Evol., 5: 407-410.

Boersma, M. 1995. The allocation of resources to reproduction in Daphnia galeata: against the odds? Ecology, 76: 1251-1261.

Bradshaw, A.D. 1965. Evolutionary significance of phenotypic plasticity in plants. Adv. Genet., 13: 115-155.

Clark, C.W. \& C.D. Harvell. 1992. Inducible defenses and the allocation of resources: a minimal model. Am. Nat., 139: 521-539.

De Meester, L. 1993. Genotype, fish-mediated chemicals, and phototactic behavior in Daphnia magna. Ecology, 74: 1467-1474.

- - - 1996. Evolutionary potential and local genetic differentiation in a phenotypically plastic trait of a cyclical parthenogen, Daphnia magna. Evolution, 50: 1293-1298. 
DeWitt, T.J. 1998. Costs and limits of phenotypic plasticity: Tests with predator-induced morphology and life history in a freshwater snail. J. Evolution. Biol., 11: 465-480.

DeWitt, T.J., A. Sih \& D.S. Wilson, 1998. Costs and limits of phenotypic plasticity. Trends Ecol. Evol., 13: 77-81.

Dicke, M. \& M.W. Sabelis. 1988. Infochemical terminology: based on cost-benefit analysis rather than origin of compounds? Funct. Ecol., 2: 131-139.

Dodson, S.I. 1988. The ecological role of chemical stimuli for the zooplankton: predator-avoidance behavior in Daphnia. Limnol. Oceanogr., 33: 1431-1439.

Dodson, S.I. \& J.E. Havel. 1988. Indirect effects: some morphological and life history responses of Daphnia pulex exposed to Notonecta undulata. Limnol. Oceanogr., 33: $1274-1285$

Gilbert, J.J. 1980. Further observations on developmental polymorphism and its evolution in the rotifer Brachionus calyciflorus. Freshwater Biol., 10: 281-294.

Gliwicz, Z.M. \& C. Guisande. 1992. Family planning in Daphnia-resistance to starvation in offspring born to mothers grown at different food levels. Oecologia, 91: 463-467.

Grant, J.W.G. \& I.A.E. Bayly. 1981. Predator induction of crests in morphs of the Daphnia carinata King complex. Limnol. Oceanogr., 26: 210-218.

Hanazato, T. 1991. Induction of development of high helmets by a Chaoborus-related chemical in Daphnia galeata. Arch. Hydrobiol., 122: 167-175.

$--\ldots$. 1992. Direct and indirect effects of low-oxygen layers on lake zooplankton communities. Arch. Hydrobiol.-Beih. Ergebn. Limnol., 35: 87-98.

Haney, J.F. \& D.J. Hall. 1973. Sugar-coated Daphnia: a preservation technique for Cladocera. Limnol. Oceanogr., 18: 331-333.

Harvell, C.D. 1984. Predator-induced defense in a marine bryozoan. Science, 224: 1357-1359.

- - - 1990. The ecology and evolution of inducible defenses. Q. Rev. Biol., 65: 323-340.

Havel, J.E. 1987. Predator-induced defenses: a review. In: W.C. Kerfoot \& A. Sih (Eds), Predation: Direct and Indirect Impacts on Aquatic Communities. University Press of New England, Hanover: 263-278

Havel, J.E. \& S.I. Dodson. 1984. Chaoborus predation on typical and spined morphs of Daphnia pulex: behavioral observation. Limnol. Oceanogr., 29: 487-494.

Hebert, P.D.N. \& D.M. Grewe. 1985. Chaoborus-induced shifts in the morphology of Daphnia ambigua. Limnol. Oceanogr., 30: 1291-1297.

Krueger, D.A. \& S.I. Dodson. 1981. Embryological induction and predation ecology in Daphnia pulex. Limnol. Oceanogr., 26: 219-223.

Littell, R.C., G.A. Milliken, W.W. Stroup \& R.D. Wolfinger (Eds). 1996. SAS System for Mixed Models. SAS Institute, Cary, NC.

Lively, C.M. 1986a. Predator-induced shell dimorphism in the acorn barnacle, Chthamalus anisopoma. Evolution, 40: 232-242.

- - - 1986b. Canalization versus developmental conversion in a spatially variable environment. Am. Nat., 128: $561-572$.

Lynch, M. 1985. Spontaneous mutations for life-history characters in an obligate parthenogen. Evolution, 38: 804-818.

Lynch, M. \& W. Gabriel. 1983. Phenotypic evolution and parthenogenesis. Am. Nat., 122: 745-764.
Milliken, G.A. \& D.E. Johnson (Eds). 1984. Analysis of Messy Data. Volume I: Designed Experiments. Lifetime Learning Publications, California.

Moran, N.A. 1992. The evolutionary maintenance of alternative phenotypes. Am. Nat., 139: 971-989.

Mort, M.A. 1986. Chaoborus predation and the function of phenotypic variation in Daphnia. Hydrobiologia, 133: 39-44.

Nilsson, P.A., C. Brönmark \& L.B. Pettersson. 1995. Benefits of a predator-induced morphology in crucian carp. Oecologia, 104: 291-296.

Padilla, D.K. \& S.C. Adolph. 1996. Plastic inducible morphologies are not always adaptive: the importance of time delays in a stochastic environment. Evol. Ecol., 10: $105-117$.

Parejko, K. \& S.I. Dodson. 1991. The evolutionary ecology of an antipredator reaction norm: Daphnia pulex and Chaoborus americanus. Evolution, 45: 1665-1674.

Riessen, H.P. 1992. Cost-benefit model for the induction of an antipredator defense. Am. Nat., 140: 349-362.

SAS Institute, 1988. SAS/STAT User's Guide, Release 6.03 Edition. SAS Institute, Inc., Cary, NC.

Scheiner, S.M. 1993. Genetics and evolution of phenotypic plasticity. Annu. Rev. Ecol. Syst., 24: 35-68.

Scheiner, S.M. \& D. Berrigan. 1998. The genetics of phenotypic plasticity. VIII. The cost of plasticity in Daphnia pulex. Evolution, 52: 368-378.

Schlichting, C.D. \& M. Pigliucci (Eds). 1998. Phenotypic Evolution: a Reaction Norm Perspective. Sinauer Associates, Sunderland, MA.

Smith, C.C. \& S.D. Fretwell. 1974. The optimal balance between size and number of offspring. Am. Nat., 108: 49-506.

Sokal, R.R. \& F.J. Rohlf (Eds). 1995. Biometry. 3rd ed. W. H. Freeman and Company, New York.

Spitze, K. 1991. Chaoborus predation and life-history evolution in Daphnia pulex: temporal pattern of population diversity, fitness, and mean life history. Evolution, 45: 82-92.

Spitze, K. \& T.D. Sadler. 1996. Evolution of a generalist genotype: multivariate analysis of the adaptiveness of phenotypic plasticity. Am. Nat., 148: 108-123.

Stibor, H. 1992. Predator induced life-history shifts in a freshwater cladoceran. Oecologia, 92: 162-165.

Taylor, B.E. \& W. Gabriel. 1992. To grow or not to grow: optimal resource allocation in Daphnia. Am. Nat., 139: 248-266.

Tessier, A.J. \& N.L. Consolatti. 1991. Resource quantity and offspring quality in Daphnia. Ecology, 72: 468-478.

Tollrian, R. 1994. Fish-kairomone induced morphological changes in Daphnia lumholtzi (Sars). Arch. Hydrobiol., 130: 69-75.

- - . 1995. Predator-induced morphological defenses: costs, life history shifts, and maternal effects in Daphnia pulex. Ecology, 76: 1691-1705.

Tollrian, R. \& S.I. Dodson. 1998. Inducible defenses in cladocera: constraints, costs, and multipredator environments. In: R. Tollrian \& C.D. Harvell (Eds), The Ecology and Evolution of Inducible Defenses. Princeton University Press, New Jersey: 177-202

Vollenweider, R.A. (Ed.) 1974. A Manual on Methods for Measuring Primary Production in Aquatic Environments. IBP Handbook 12, Blackwell, Oxford: 255 pp. 